\title{
Melanoma of unknown primary origin metastasizing to the ovary and breast: Case report and review of literature
}

\author{
Shuang Niu, MD, PhD ${ }^{1}$, Long Li, MD, PhD², and Yen Peng, MD, PhD ${ }^{1, *}$ \\ Department of Pathology, University of Texas Southwestern Medical Center, Dallas, Texas, USA \\ 2 Department of Pathology and Genomic Medicine, Houston Methodist Hospital, Houston, Texas, USA
}

\begin{abstract}
Malignant melanoma (MM) simultaneously involving the ovary and breast is a rare manifestation of the disease. We report a case of amelanotic MM of unknown primary origin manifesting as a large ovarian solid mass at its first clinical presentation. A 46-year-old woman presented with a two week history of lower abdominal pain. Imaging studies revealed an $18 \mathrm{~cm}$ right ovarian mass and a $1.1 \mathrm{~cm}$ left breast mass. The ovarian mass was first removed and it showed smooth capsule. Histologically, the tumor exhibited predominantly solid and nested growth patterns with scattered follicle-like spaces. The cells had large atypical nuclei with prominent nucleoli, and brisk mitotic activity. No melanin pigment was identified. No evidence of mature teratoma in the ovary was found. Immunohistochemically, the tumor was diffusely positive for SOX10, S-100, HMB-45, Melan-A and WT-1, and negative for cytokeratins, PAX-8, ER, PR, CD45, SALL4, and inhibin. Biopsy of the breast lesion showed a poorly differentiated malignancy with identical morphology. A diagnosis of metastatic melanoma to the ovary and breast was established. Thorough physical examination and imaging study revealed no evidence of cutaneous, uveal, or mucosal lesions. Probably, a primary MM was occult or had regressed. Subsequent molecular test for BRAF V600E mutation in the tumor was positive. The patient rapidly developed widespread metastasis. Immunotherapy was initiated; she had excellent response with significant tumor regression at her six month follow up visit. This case represents an example that making correct and timely diagnosis of metastatic melanoma followed by prompt targeted therapy significantly improved the patient's clinical outcome.
\end{abstract}

Keywords: metastatic melanoma; ovary; breast; BRAF V600E mutation

\section{Introduction}

Cancers from outside female reproductive tract often metastasize to the ovary and account for less than $10 \%$ of all ovarian malignant neoplasms [1, 2]. The most common origins are colon, appendix, stomach, and breast [1-3]. Malignant melanoma (MM) is a tumor of high metastatic potential and frequently spreads to the skin, lymph nodes, lung, brain, liver and bone [4]. Ovarian involvement by $\mathrm{MM}$ is uncommon and usually seen in patients with disseminated disease [5]. MM clinically manifesting as an ovarian mass is very rare and can pose significant diagnostic difficulties. Here we report a case of amelanotic malignant melanoma metastasizing to the ovaries and breast in a patient without previous history of melanoma or resected nevus. We emphasize on the diagnostic features of this case, and highlight the importance of taking melanoma into consideration even in the absence of relevant history.

\section{Case report}

A 46-year-old multiparous Caucasian female presented with two weeks history of lower abdominal pain and increased abdominal girth. Her medical history included viral hepatitis and alcohol abuse while her gynecological history was unremarkable. She also experienced malaise, decreased appetite, early satiety, and low-grade fever. Physical exam showed distended abdomen with a firm irregular mass in the left lower quadrant. Transvaginal ultrasound revealed a large solid and cystic mass of the right adnexa. Later, smaller nodules were found in the left ovary, breast, lung, peritoneum by the preoperative computed topography (CT) scan, all concerning for malignancies. The tumor marker CA125 was elevated at 560 units/mL (reference range < 35), whereas CA19-9 and CEA were within normal limits. PAP smear and endometrial biopsy showed no abnormalities. Bilateral salpingo-

*Corresponding author: Yan Peng, MD., PhD., Professor of Pathology, University of Texas Southwestern Medical Center, 5323 Harry Hines Blvd, Dallas, Texas 75390, USA. Tel.: (214) 633-6347; FAX: (214) 633-8817; Email: Yan.Peng@utsouthwestern.edu

Shuang Niu and Long Li: equal contribution to the work

Received 12 October 2018 Revised 11 January 2019 Accepted 25 January 2019 Published 31 January 2019

Citation: Niu S, Li L, Peng Y. Melanoma of unknown primary origin metastasizing to the ovary and breast: Case report and review of literature. J Mod Hum Pathol. 2019; 4(1):1-5. DOI: 10.14312/2397-6845.2019-1

Copyright: (c) 2019 Yan Peng, et al. Published by NobleResearch Publishers. This is an open-access article distributed under the terms of the Creative Commons Attribution License, which permits unrestricted use, distribution and reproduction in any medium, provided the original author and source are credited. 
oophorectomy and omentectomy were performed. The 18 $\mathrm{cm}$ right ovarian mass had a smooth surface and a solid/ cystic parenchyma (Figure 1).

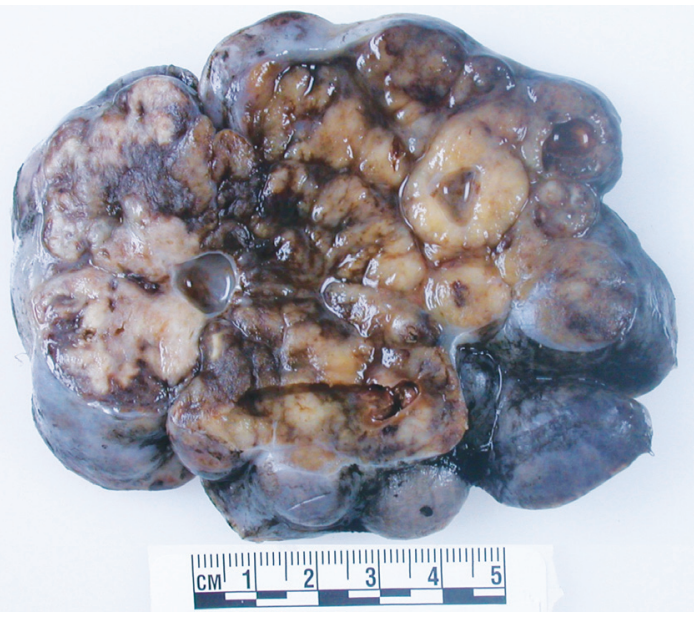

Figure 1 Grossly, the tumor had a smooth, cerebriform surface and a solid and cystic parenchyma.
Frozen section showed the tumor was composed of solid sheets and nodules of malignant cells with numerous mitotic figures and apoptotic bodies. No apparent epithelioid differentiation or psammoma bodies were identified. A diagnosis of poorly differentiated carcinoma was rendered intraoperatively (Figure 2a). On permanent sections, the tumor contained pseudoglandular areas associated with hemorrhage, and scattered follicle-like spaces with proteinaceous fluid (Figure 2b, c). Under high magnification, the tumor cells were relatively monotonous with indistinct cell borders, scant eosinophilic to clear cytoplasm, round to ovoid nuclear contour, and fine chromatins. The nucleoli were visible in many cells and were prominent in some. Longitudinal nuclear grooves were occasionally present (Figure 2d). Brown, coarse pigments were in clusters, especially in the areas surrounding the hemorrhage; the pigments were considered hemosiderin materials (Figure 2d). The tumor lacked spindle cell component or sarcomatoid differentiation. No endometriosis, mature teratoma, or in situ carcinoma was found in the background ovary or the fallopian tubes.

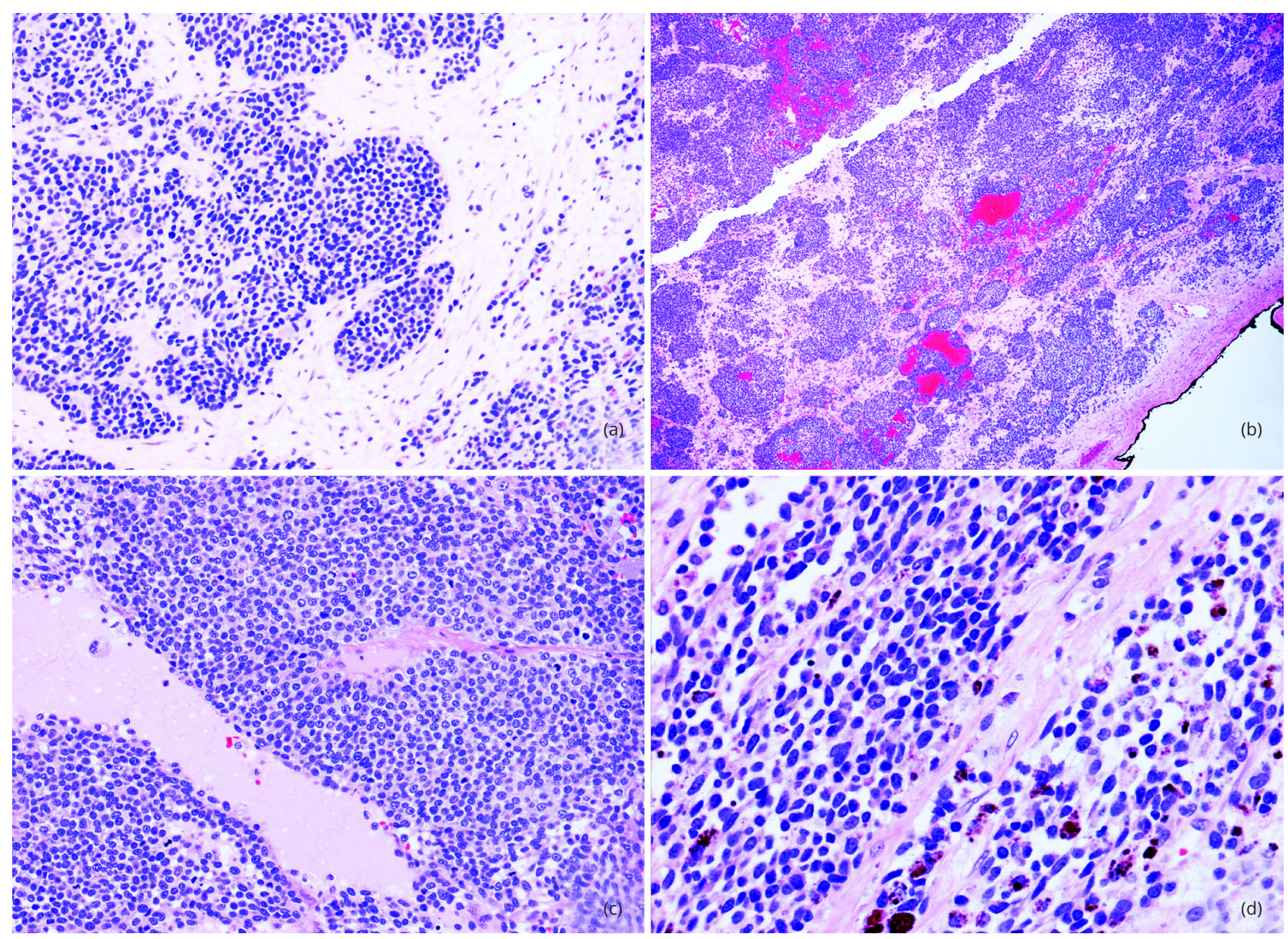

Figure 2 Frozen section showed the tumor was composed of solid sheets and nodules of malignant cells (a). Tumor contained pseudoglandular areas associated with hemorrhage (b), and scattered follicle-like spaces with proteinaceous fluid (c). The tumor cells were relatively monotonous with indistinct cell borders, scant eosinophilic to clear cytoplasm, round to ovoid nuclear contour, and fine chromatins. The nucleoli were visible in many cells and were prominent in some (d). Brown, chunky pigments were in clusters, especially in the areas surrounding the hemorrhage.

Sections from the left ovary, omental and peritoneal nodules showed tumor of the same morphology. Immunohistochemically, the neoplastic cells were negative for cytokeratins AE1/AE3 (Figure 3a), Cam5.2, CK5/6, CK7, CK20 and CK18, PAX-8 (Figure 3b), estrogen receptor (ER), progestin receptor (PR), CD10, p16, CD45, SALL4, calretinin, 
inhibin, desmin and myogenin. P53 stain showed nonmutant, patchy pattern. CD99 showed diffuse membranous stain with heterogeneous intensity. Vimentin and WT-1 were strongly positive throughout. Neuroendocrine marker CD56 was focally weak positive. Chromogranin was weak positive; TTF-1 and synaptophysin are negative. Melanocytic markers, S-100 (Figure 3c), HMB-45 (Figure 3d) and SOX10 were strongly positive and Melan-A was weakly positive. The pigments were Melan-A negative hemosiderin depositions.
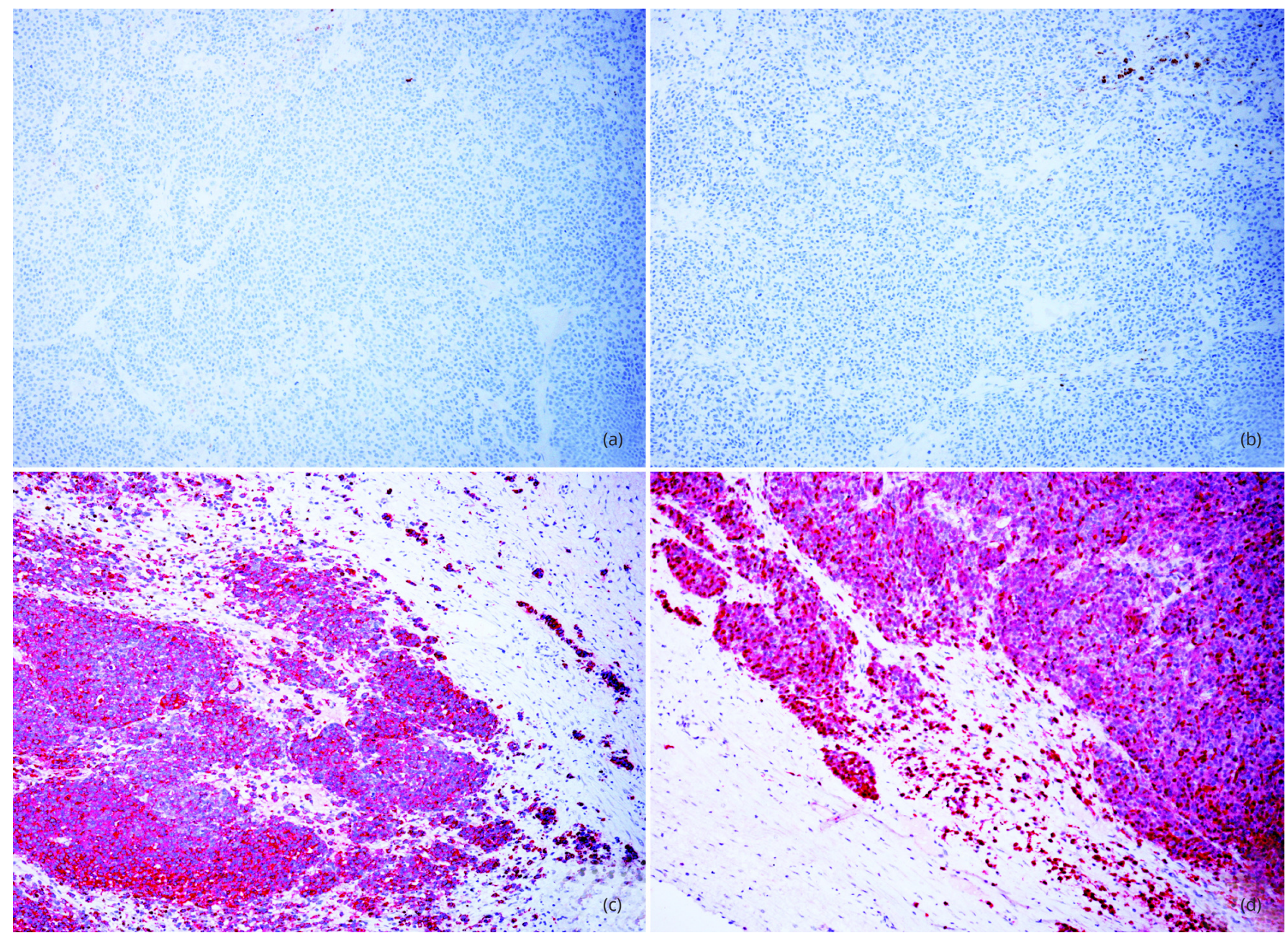

Figure 3 The tumor cells are negative for AE1/AE3 (a), PAX-8 (b), and positive for S-100 (c) and HMB-45 (d).

A histological diagnosis of malignant melanoma was made. Subsequently, molecular test for BRAF ${ }^{\mathrm{V} 600 \mathrm{E}}$ mutation was performed using the formalin fixed, paraffin embedded tumor tissue by Pyrosequencing, and the result was positive. Fluorescence in situ hybridization (FISH) test with probe for EWSR1 rearrangement was negative, excluding the possibility of melanoma of soft parts. The breast mass was then biopsied with a finding of metastatic melanoma. Postoperative imaging showed the patient rapidly developed widespread metastatic disease to multiple organs including the brain, heart, liver, kidney, soft tissue, and lymph nodes. Immunotherapy with Ipilimumab (CTLA4 monoclonal antibody) and Nivolumab (anti-PD-1 antibody) was initiated. She had excellent response to the therapy with significant tumor regression, and was alive at six months follow up (BRAF inhibitors were reserved as second line therapy).

\section{Discussion}

Metastatic cancers account for less than 10\% of all ovarian malignant neoplasms. The most common origins are gynecologic tract, gastrointestinal tract, and breast. Melanomas are very aggressive tumors with high metastatic potential. In patients died due to metastatic melanomas, about 20\% individuals had ovarian involvement based on autopsy examination [6]. The clinical significance in this scenario is minimal since it represents a part of widely disseminated disease and does not affect the clinical management. In contrast, MM clinically presenting as an ovarian mass is exceedingly rare, and can pose major diagnostic difficulties. Two major case series of ovarian MM provided detailed insight to these rare cases $[7,8]$. Individual case reports were also found in the literature [6, 9-13]. Over $60 \%$ of these patients had known histories of cutaneous melanomas or pigmented lesions. The most frequent primary tumor sites were the extremities [8]. The interval between previous diagnoses to the discovery of ovarian tumors ranged from 1 month to 13 years $[7,11]$. Concurrent extra-ovarian metastases were commonly found in the pelvis, peritoneal cavity, and lymph nodes. In contrast to other types of metastatic tumors, the ovarian metastases from melanoma were mostly unilateral [8]. 
Almost all cases had smooth intact ovarian surface. The average tumor size was $10 \mathrm{~cm}$. Pigmentation was only seen in about $30-35 \%$ of malignant melanomas but as could also be seen in steroid tumors [7, 8]. Nodular and diffuse growth patterns were the most common architecture. Nested pattern, if present, was indicative of MM. The neoplastic cells were most commonly epithelioid with abundant eosinophilic or clear cytoplasm mimicking dysgerminomas. Pseudo-nuclear inclusions and prominent nucleoli were often present. The monotonous appearance and longitudinal nuclear grooves may give an impression of adult granulosa cell tumor (GCT). The presence of follicle-like structures may mimic juvenile granulosa cell tumor or ovarian small cell carcinoma of hypercalcemic type. Pseudoglandular areas due to hemorrhage may resemble a primary ovarian carcinoma. Less commonly, the neoplastic cells are small with scant cytoplasm, and thus the differential diagnoses of small round cell tumors such as small cell carcinoma, lymphoma, primitive neuroectodermal tumor (PNET), desmoplastic small round cell tumor, or synovial sarcoma might be entertained [14]. Spindle cells were less frequent and usually were mixed with epithelioid areas. Immunohistochemistry with S-100, HMB-45, and Melan-A are all helpful in establishing a diagnosis [15]. S-100 has the highest sensitivity compared to the other markers. However, it needs to be noted that in distinction with ovarian sex cord stromal tumors, one should not rely on any single marker alone. Ovarian sex cord stromal tumors, especially granulosa cell tumors, are often positive for S-100 and Melan-A [8, 16, 17], while melanomas can be focally or weakly positive for inhibin and calretinin [8]. Additionally, sex cord stromal tumor are often positive for CD56 and WT-1, further confounding the matter in the current case. HMB-45 is less sensitive than S-100, but is widely regarded as the most specific melanoma marker and is negative in GCTs [15]. Use of an IHC panel and interpreting the tumor's IHC profile in the context of clinical, gross, and microscopic features is recommended.

Once the diagnosis of melanoma is established, the origin needs to be identified if possible. According to one study of data from National Cancer Data Base, the percentages of cutaneous, mucosal, uveal, or unknown primary melanomas are $91.2 \%, 5.2 \%, 1.3 \%$, and $2.2 \%$, respectively [18]. Several possible explanations exist when a primary lesion cannot be detected in a metastatic melanoma. The first one is regression of primary melanoma, which occurs in about 2-9\% cutaneous melanomas [7]. This is also supported by individual case reports [19]. The second possibility is small occult primary lesions that were left unnoticed. For an ovarian melanoma of unknown primary, one additional possibility is bona fide primary melanoma arising from a mature cystic teratoma. Primary ovarian melanomas often affect postmenopausal women [10], while metastatic MM affect younger women due to the higher blood flow to the premenopausal ovary [20]. The incidence of primary ovarian melanomas is much lower than that of metastatic MM [5, 21]. The presence of associated teratoma is generally required to establish this diagnosis $[5,22]$. Therefore, the distinction usually can be made by careful examination of the specimen.
Successful diagnosis of ovarian MM of unknown primary heavily relies on a pathologist's experience and awareness of the entity. The clinical presentations, imaging characteristics, tumor markers, are largely non-specific. It is reported that MRI may aid the detection of melanin producing lesion yet the majority of ovarian melanomas are amelanotic [13]. Without obvious clinical history as a hint, it can be easily misdiagnosed as sex cord stromal tumor or poorly differentiated carcinoma, leading to different therapeutic strategies. As mentioned above, isolated metastasis to the ovary from MM is exceedingly rare. Once a diagnosis of ovarian melanoma is reached by a pathologist, it is crucial to alert the clinician to search for potentially more disseminated disease. Search of an occult primary may help to illustrate a complete clinical picture. However, it carries limited value since in the presence of distant metastasis the survival is similar regardless of the origins [23]. Surgery is a suitable approach for resectable tumors $[2,11]$. The role of postoperative chemotherapy is controversial but has been shown to be beneficial by some studies [9-11]. BRAF mutation is present in about $50 \%$ of metastatic MM of unknown primary tumors [24]. Although earlier data found the mutation status has no impact on the survival, the emerging immunotherapies with BRAF inhibitors, MEK inhibitors, CTLA4 and PD1 inhibitors may improve the outcome of these advanced melanoma patients in the near future $[25,26]$.

\section{Conclusion}

In summary, we report a case of amelanotic malignant melanoma of unknown origin clinically manifesting as an ovarian mass. The absence of clinical history of melanoma made the diagnosis of this case very challenging. Yet the successful pathological diagnosis is important and has significant clinical impacts. In any given tumor that does not closely resemble primary ovarian cancers, it is important to take melanoma into consideration and perform a panel of immunohistochemistry stains to rule out this possibility. This is especially important due to amelanotic nature of most ovarian melanomas. It is also important to prompt a thoroughly search by the clinician for concurrent extraovarian metastasis since isolated ovarian involvement is very rare. Finally, characterizing the ovarian metastatic tumor molecularly is an intrinsic part of patient care in the era of personalized medicine. Based on BRAF mutation status, new treatment modalities such as BRAF, CTLA4, PD1 inhibitors can be initiated in combination or alone for advanced melanoma patients.

\section{Conflicts of interest}

Authors declare no conflicts of interest.

\section{References}

[1] Moore RG, Chung M, Granai CO, Gajewski W, Steinhoff MM. Incidence of metastasis to the ovaries from nongenital tract primary tumors. Gynecol Oncol. 2004; 93(1):87-91.

[2] Ayhan, A, Guvenal T, Salman MC, Ozyuncu O, Sakinci M, et al. The role of cytoreductive surgery in nongenital cancers metastatic to the ovaries. Gynecol Oncol. 2005; 98(2):235-241.

[3] Alvarado-Cabrero I, Rodríguez-Gómez A, Castelan-Pedraza J, ValenciaCedillo R. Metastatic ovarian tumors: A clinicopathologic study of 150 cases. Anal Quant Cytopathol Histpathol. 2013; 35(5):241-248. 
[4] Buzaid, AC, Agarwala SS, Hauschild A, Atkins M. Algorithm for the management of metastatic cutaneous melanoma. Chin Clin Oncol. $2014 ; 3(3): 32$.

[5] Sbitti, Y, Fadoukhair Z, Kadiri H, Oukabli M, Essaidi I, et al. Diagnostic challenge for ovarian malignant melanoma in premenopausal women: Primary or metastatic? World J Surg Oncol. 2011; 9:65.

[6] Piura B, Kedar I, Ariad S, Meirovitz M, Yanai-Inbar I. Malignant melanoma metastatic to the ovary. Gynecol Oncol. 1998; 68(2):201-205.

[7] Young RH, Scully RE. Malignant melanoma metastatic to the ovary. A clinicopathologic analysis of 20 cases. Am J Surg Pathol. 1991; 15(9):849-860.

[8] Gupta D, Deavers MT, Silva EG, Malpica A. Malignant melanoma involving the ovary: A clinicopathologic and immunohistochemical study of 23 cases. Am J Surg Pathol. 2004; 28(6):771-780.

[9] Boutis A, Valeri R, Korantzis I, Valoukas D, Andronikidis I, et al. Delayed malignant melanoma recurrence simulating primary ovarian cancer: case report. World J Surg Oncol. 2008; 6:124.

[10] Ueng SH, Pinto MM, Alvarado-Cabrero I, Lee LY, Tavassoli FA. Ovarian malignant melanoma: A clinicopathologic study of 5 cases. Int J Surg Pathol. 2010. 18(3):184-192.

[11] Oliver R, Dasgupta C, Coker A, Al-Okati D, Weekes AR. Ovarian malignant melanoma: Unusual presentation of a solitary metastasis. Gynecol Oncol. 2005; 99(2):412-414.

[12] Pietzner K, Noske A, Cho $\mathrm{CH}$, Kiecker F, SehouliJ. Amelanotic metastasis of melanoma mimicking ovarian cancer: A case report and review of the literature. Anticancer Res. 2008; 28(1B):563-566.

[13] Moselhi M, Spencer J, Lane G. Malignant melanoma metastatic to the ovary: Presentation and radiological characteristics. Gynecol Oncol. 1998; 69(2):165-168

[14] McCluggage WG. Ovarian neoplasms composed of small round cells: A review. Adv Anat Pathol. 2004; 11(6):288-296.

[15] McCluggage WG, Young YH. Immunohistochemistry as a diagnostic aid in the evaluation of ovarian tumors. Semin Diagn Pathol. 2005; 22(1):3-32.

[16] Stewart CJ, Nandini CL, Richmond JA. Value of A103 (melan-A) immunostaining in the differential diagnosis of ovarian sex cord stromal tumours. J Clin Pathol. 2000; 53(3):206-211.

[17] Costa MJ, DeRose PB, Roth LM, Brescia RJ, Zaloudek Cl, et al. Immunohistochemical phenotype of ovarian granulosa cell tumors: Absence of epithelial membrane antigen has diagnostic value. Hum Pathol. 1994; 25(1):60-66.

[18] Chang AE, Karnell LH, Menck HR. The National Cancer Data Base report on cutaneous and noncutaneous melanoma: A summary of 84,836 cases from the past decade. The American College of Surgeons Commission on Cancer and the American Cancer Society. Cancer. 1998; 83(8):1664-1678.

[19] Martinelli G, Tapparelli E, Merz R, Aldovini D, Zumiani G. Case of bilateral ovarian metastasis from regressed melanoma. Eur J Gynaecol Oncol. 1984; 5(2):150-153.

[20] Jeremic K, Berisavac M, Argirović R, Gojnić M, Bosković V, et al. Solitary ovarian metastasis from cutaneous melanoma-case report. Eur J Gynaecol Oncol. 2006; 27(4):443-444.

[21] Kudva R, Ayachit GS, Ayachit A. Malignant melanoma arising in an ovarian mature cystic teratoma - a rare entity. J Clin Diagn Res. 2015; 9(4):Ed14-16.

[22] McCluggage WG, Wilkinson N. Metastatic neoplasms involving the ovary: A review with an emphasis on morphological and immunohistochemical features. Histopathology. 2005; 47(3):231-247.

[23] Kuk D, Shoushtari AN, Barker CA, Panageas KS, Munhoz RR, et al. Prognosis of mucosal, uveal, acral, nonacral cutaneous, and unknown primary melanoma from the time of first metastasis. Oncologist. 2016; 21(7):848-854.

[24] Heppt MV, Tietze JK, Reinholz M, Rahimi F, Jung A, et al. Disease kinetics but not disease burden is relevant for survival in melanoma of unknown primary tumor. Discov Med. 2015; 20(110):231-237.

[25] Franklin C, Livingstone E, Roesch A, Schilling B, Schadendorf D. Immunotherapy in melanoma: Recent advances and future directions. Eur J Surg Oncol. 2017; 43(3):604-611.

[26] Simeone E, Grimaldi AM, Festino L, Vanella V, Palla M, et al. Combination treatment of patients with BRAF-mutant melanoma: A new standard of care. BioDrugs. 2017; 31(1):51-61. 